\title{
HHV6 infection in a 32days old baby, its presentation and management
}

\begin{abstract}
Background: Human herpes virus 6 (HHV-6), commonly known to cause the childhood disease roseola and nonspecific viral illness, has been known to cause serious neurological complications including fever induced seizures and encephalitis with possible long term sequel especially in immune compromised patients.

Purposes: To report a rare case of HHV6 meningitis in a 32days old baby boy and the challenging therapeutic approach.

Case summary: A case of 32days old baby boy presented with one episode of high grade fever and bulging anterior fontanel in the absence of skin rashes. Blood tests were normal and lumbar puncture done at admission revealed lymphocytic pleocytsis secondary to acute HHV6 confirmed by CSF PCR. Even though lacked data about appropriate treatment in this age group, our patient received Ganciclovir IV for 14days and showed clinical improvement.

Conclusion: Treatment of HHV6 meningitis with Ganciclovir is recommended for immune compromised patients, including immune deficient patients, and those receiving chemotherapy or prolonged course of steroid. However, literature does not support any specific treatment for immune competent children. No data or clear recommendations for the treatment of neonates with HHV6 meningitis. Should neonate be considered like immune compromised host and be treated with Ganciclovir or should they only receive supportive treatment? More studies are required to have an evidence based antiviral treatment in this age group.
\end{abstract}

Keywords: HHV6, meningitis, Ganciclovir
Volume 8 Issue 6 - 2018

\author{
El Ahmar Malak,' Srour Sara,' Hanna pielly,' \\ Beih Farah,' Sacy Robert, ${ }^{2}$ Ibrahim $\mathrm{Nada}^{3}$ \\ 'Department of pediatric, Lebanese University, Lebanon \\ ${ }^{2}$ Professor at the Balamand University, department of pediatric \\ and NICU at St George's hospital, Lebanon \\ ${ }^{3}$ Pediatrician \& Department of Pediatric Infectious Diseases, \\ Lebanon
}

\begin{abstract}
Correspondence: Robert Sacy, Professor at the Balamand University, Department of pediatric and NICU at St George's hospital, Lebanon, Email robartgsacy@gmail.com
\end{abstract}

Received: September 30, 2018 | Published: November 16 , 2018

\section{Abbreviations: HHV6, human herpes simplex 6}

\section{Introduction}

HHV-6 is the sixth members of the beta herpes subfamily and is a DNA virus that is expressed as 2 closely related variants, A and B. ${ }^{1}$ among the human herpes viruses HHV6 and 7 are most closely related to Cytomegalovirus. Only 20\% of infected children will develop roseola infantum and the other $80 \%$ will only have undifferentiated febrile illness without rash or localized signs. HHV-6B is more common and frequently demonstrated to be the causative agent of roseola infantum in healthy infants. ${ }^{2}$ By the age of 3years, most of children have contracted the infection and manifested as a nonspecific febrile illness. ${ }^{1}$ However HHV-6 infection may be complicated by seizure, and more serious central nervous system complications, probably due to its neurotropic properties, including encephalopathy , aseptic meningitis , meningo encephalitis, hemiplegia and multiple sclerosis and other long term neurological sequel., ${ }^{3,4}$

\section{Case report}

A 32days old baby boy, presented to our emergency department for his first episode of high grade fever reaching 39 degrees associated with one day history of cough. The mother noted good activity and adequate PO intake of the baby. There were no associated gastrointestinal symptoms (diarrhea or vomiting), no irritability, no abnormal movements, no skin rash (petechial purpura), no mottled skin, no hypo activity, and no cyanosis. He is a full term baby, born by normal vaginal delivery, no ICN admission, previously healthy, to a 25years old healthy mother, moderately smoker (mainly Hubble bubble), well followed pregnancy, smooth course, none complicated, GBS and TORCH status not available.

G6P3A3: She had three previous abortions all in the first trimester of pregnancy without obvious cause. There is no consanguinity between the parents. Currently baby is taken vitamin D (400IU) supplementation only. He is breastfed and bottle fed with regular for age cow's milk. He received his first vaccination at birth (hepatitis B). The baby also has 2 healthy siblings, a 3yo sister and 1.5 yo brother, with a recent history of isolated fever without skin rash or headache.

Physical exam: upon arrival to ED, the baby is active tonic playful well appearing febrile, 38.9 degrees, other vital signs were normal. The physical exam was unremarkable except for an appreciable bulging anterior fontanel. No visible skin rash and no irritability were noted.

Laboratory: Immediate full sepsis workup was done, starting with complete blood test, urine analysis, stool analysis, Chest XRAY, LP and cultures. Blood tests were unremarkable with WBC of 9000 and neutrophils $24 \%$ and negative CRP. Examination of his cerebrospinal fluid performed on the day of presentation revealed CSF-WBC 265 and RBC 52 with $82 \%$ lymphocytic and $18 \%$ segmented. CSF-protein was $1.04 \mathrm{~g} / \mathrm{L}$ and CSF-glucose was $32 \mathrm{mg} / \mathrm{dL}$; both within normal range. Urine analysis is negative, stool analysis revealed neither adenovirus nor rotavirus. Chest X-ray showed no abnormalities. Later on, urine, stool and blood culture showed no growth after 5 days. CSF culture also showed no growth of any pathogens. CSF 
sent for real time PCR using the multiplex PCR to detect multiple viral pathogens. PCR human herpes virus 6 in the CSF was positive. However PCR for all other viral pathogens were negative including: EBV, CMV, adenovirus, HSV1 and 2, VZV, HHV7, and parvovirus. During his stay in the hospital, the baby was stable hemodynamic ally. The fever improved in the first 24hours. He started immediately on IV Ganciclovir continued for a total of 14days. His peripheral white count ranged from 6000 to 9900 and his liver enzymes were always normal. Ultrasound brain was also done and revealed no parenchymal abnormalities.

\section{Discussion}

Primary HHV-6 infection manifest in different ways. HHV-6 infection is more often associated with a nonspecific febrile illness between 6 months and 2years of life. ${ }^{1}$ Most commonly, symptoms include fever, and rash. ${ }^{1}$ HHV-6 is most likely transmitted via the saliva of healthy individuals and enters the body via a mucosal surface. After an incubation period of 10 to 15 days, the illness start by a prodromal of mild rhinorrhea, sore throat, and conjunctiva redness, followed by a high fever. ${ }^{5}$ Then, fewdays later, the fever abates and the rash of roseola begin as small, erythematous, raised papules on the trunk that spread to the neck and extremities lasting 1 to 3 days. ${ }^{4}$ HHV-6 infection is frequently associated with the first manifestation of benign febrile seizures in childhood. While these seizures are more commonly related to the febrile response to HHV-6 infection, there is a subset of patients who have direct central nervous system HHV-6 infection accounting for the seizures. The frequency of such cases of encephalitis and meningo encephalitis is unclear due to the lack of baseline testing for HHV-6 in children with simple and complex febrile seizures. Additionally, the exact role that HHV-6 plays in neurological infections is not well understood. Neurologic manifestations of the disease can include generalized, repetitive, and prolonged seizures. Ataxia, weakness, hemiplegia, and disturbances in consciousness can also occur. Complications such as meningo encephalitis and encephalopathy can be severe and lead to poor outcomes.

Conclusive laboratory diagnosis of HHV-6 encephalitis may sometimes be difficult. To investigate whether HHV-6 is an etiological agent of encephalitis, the CSF should be examined for evidence of virus infection by determining the presence of viral sequence using PCR. Elevated serum IgMs may be suggestive of recent infection and possible post-infectious encephalitis, but also may not be the direct cause of CNS disease. Even positive blood PCRs for HHV-6 does not confirm the presence of this agent in the CNS. To be certain of the diagnosis of active CNS infection with HHV-6, CSF sampling is required, and positive CSF PCRs indicate active CNS infection with HHV-6. While CSF PCR is definitive, serum IG M and PCR can be used to help assure the diagnosis prior to starting toxic therapy. ${ }^{1}$

Patients with primary HHV-6 infection are typically mild and usually require only symptomatic treatment such as antipyretics and hydration with no other specific antiviral treatment. Currently there are no approved therapies for the treatment of HHV6 encephalitis. ${ }^{6}$ Antivirals such as ganciclovir, foscarnet, and cidofovir are usually reserved for immune compromised patients or those with CNS involvement. ${ }^{4}$ These drugs reported success only in a few studies and case reports. Despite the lack of controlled data, Foscarnet and ganciclovir (valganciclovir) are recommended as the first line of treatment, cidofovir is the second. The International Herpes Management Forum recommends the use of foscarnet and ganciclovir, either individually or combined, to treat progressive neurologic disease due to HHV-6 infection. ${ }^{6}$ Cidofovir, an acyclic nucleoside phosphonate, has been found to be more inhibitory than ganciclovir or foscarnet in vitro. ${ }^{7}$ However, it is unclear how well it crosses the blood brain barrier and has associated drug toxicities, so at this time it is still not recommended for treating HHV-6 related infections. ${ }^{8}$ Ganciclovir high dose of $18 \mathrm{mg} / \mathrm{kg} /$ day or foscarnet $60 \mathrm{mg} / \mathrm{kg}$ twice a day was suggested for the treatment of a CNS HHV-6 infection. ${ }^{5}$ The antiviral previously described are effective against active HHV-6 infections, but the indications for treatment, as well as the conditions and duration of drug administration, are not formally approved to date. ${ }^{8}$ New antiviral drugs with wider range of activity (e.g., brincidofovir) and their combination with immunotherapy (e.g., IVIG) or other types of therapy are required to combat roseolovirus-associated CNS complications on a very carefully balanced, individual basis. ${ }^{5}$ Numerous pending questions about HHV-6 infection should stimulate future research works on the pathophysiology, diagnosis, and therapy of this virus.

\section{Conclusion}

The scale of the contribution of Roseolovirus infection to diverse neurological diseases has not been appreciated in the past, but such cases must now be fully investigated for these viruses. ${ }^{5}$ Improved knowledge of the spectrum of disease and neurologic outcomes for HHV-6 encephalitis will also help to guide antiviral therapy decisions based on clinical severity. ${ }^{1}$ The range of CNS manifestations ascribed to these viruses includes asymptomatic infection, febrile convulsions, seizure disorders, meningitis, meningoencephalitis, facial palsy, vestibular neuritis, demyelinating disorders, hemiplegia, and, rarely, fatal encephalitis. ${ }^{9}$ For nowadays, it is highly unlikely that any drug will be developed specifically for the treatment of HHV-6 infections. ${ }^{7}$ As long as the causative role of HHV-6 in neurological diseases with significant morbidity is not well established, the development of specific treatment for this virus will remain a relatively low priority. ${ }^{7}$ In conclusion, HHV-6 may occasionally cause meningitis in young infants, especially in newborn period. On the other hand, further studies are required to define the role of HHV-6 and HHV-7 in neurologic disorders such as meningitis or encephalitis in immunocompetent hosts. New emerging studies should focus on finding a safe and effective broad spectrum antiviral drug and duration of treatment for the appropriate management of roseola associated comorbidities.

\section{Declarations}

\section{Ethics approval and consent to participate}

The submitted manuscript involves a case report of a human and has been approved by the ethics committee of the Lebanese University.

\section{Authors' contributions}

All co authors contributed equally to this manuscript. Authors confirm that that the manuscript was revised by all the named authors and approved. There is no other person who satisfied the criteria of authorship who is not listed. The order of authors listed is also approved by all the contributed authors.

\section{Acknowledgements}

This case was supported by the Lebanese university, Zahraa University Hospital. Authors would like to show them some gratitude. 


\section{Competing interests}

The authors have no conflict of interest.

\section{Funds}

This research didn't receive any grant from funding agencies in the public or commercial sections.

\section{References}

1. Thrasher AN, Chusid MJ, Jacobson RD, et al. HHV-6 Infection in a Case of an Infant with Fever, Seizures, and Shock. WMJ. 2010;109(3):153-155.

2. Hukin J1, Farrell K, MacWilliam LM, et al. Case-control study of primary human herpesvirus 6 infection in children with febrile seizures. Pediatrics. 1998;101(2):E3.

3. David W Kimberlin, Richard J Whitley. Human herpesvirus-6 neurologic implications of a newly-described viral pathogen. Journal of neurovirology. 1998;4(5):474-485.
4. O'Grady JS. Fifth and sixth diseases: more than a fever and a rash. J Fam Pract. 2014;63(10):E1-E5.

5. Ongrádi J, Ablashi DV, Yoshikawa T, et al. Roseolovirus-associated encephalitis in immuno competent and immuno compromised individuals. J Neurovirol. 2017;23(1):1-19.

6. Cécile Tremblay, Michael T Brady. Human Herpesvirus 6 infection in children: Clinical manifestation, diagnosis and treatment. 2018.

7. Prichard MN, Whitley RJ. The Development of New Therapies for Human Herpesvirus 6. Curr Opin Virol. 2014;9:148-153.

8. Agut H, Bonnafous P, Gautheret-Dejean A. Laboratory and Clinical Aspects of Human Herpesvirus 6 Infection. Clin Microbiol Rev. 2015;28(2):313-335.

9. Ansari A, Li S, Abzug MJ, et al. Human Herpes viruses 6 and 7 and Central Nervous System Infection in Children. Emerg Infect Dis. 2004;10(8):1450-1454. 